\title{
S9 Figure.
}

\section{Calibration set size comparison -Halle 2015 - Within traits}

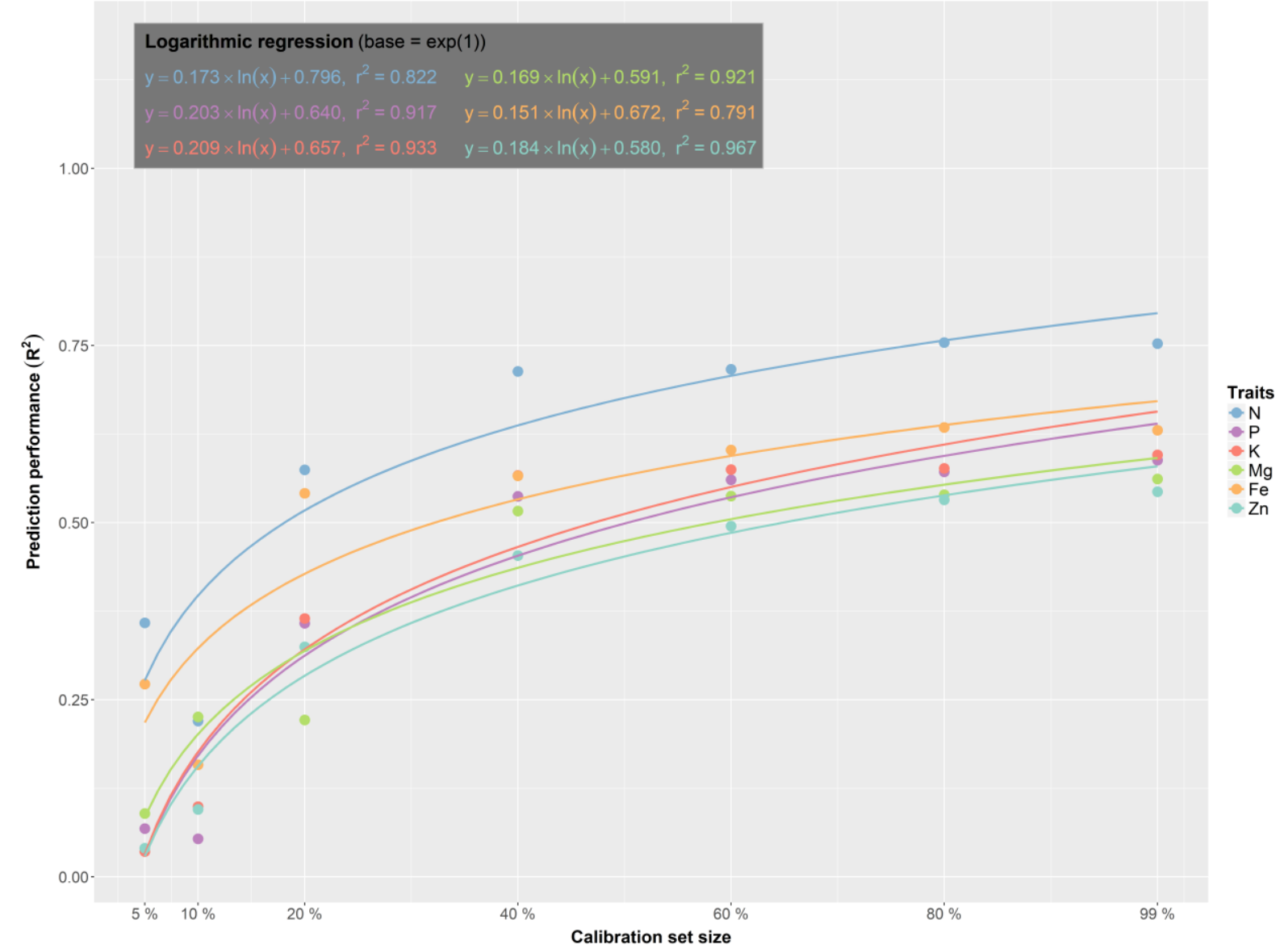

S9 Figure. Impact of calibration set size on prediction performance $\left(R^{2}\right)$ in Halle 2015 for each of the six nutrient traits (N, P, K, Mg, Fe \& Zn). A logarithmic function was fitted, which indicates the gain in prediction performance $\left(\mathrm{R}^{2}\right)$ with increasing calibration set sizes. The formulas of the six functions are shown in the upper left corner. The color of the dots and lines represents the traits. 